\title{
Prediction from Expert Demonstrations for Safe Tele-surgery
}

\author{
Ashirwad Chowriappa ${ }^{1,2} \quad$ Raul Wirz ${ }^{3} \quad$ Aditya Reddy Ashammagari ${ }^{3} \quad$ Yong Won Seo $^{3}$ \\ ${ }^{1}$ Department of Urology, Roswell Park Cancer Institute, Buffalo, New York, USA \\ ${ }^{2}$ Department of Computer Science and Engineering, The State University of New York, Buffalo, New York, USA \\ ${ }^{3}$ Department of Mechanical and Aerospace Engineering, The State University of New York, Buffalo, New York, USA
}

\begin{abstract}
In this paper, we propose a new prediction from expert demonstration (PED) methodology to improve reliability and safety in tele-surgery. Data was collected from expert (clinician) demonstrations for the procedure of trocar insertion. We encoded a set of force, torque and penetration trajectories by using a Gaussian mixture model (GMM). A generalization of these profiles and associated parameters were retrieved by Gaussian mixture regression (GMR). We validated the proposed methodology for tele-robotic placement of the trocar in two stages. First, we tested the efficacy of the proposed PED approach for handling transmission error and latency. Our results showed that for the average case ( $12 \%$ packet error and $10 \%$ loss of packet), a $58.8 \%$ improvement in performance was obtained in comparison to using an extended Kalman filter. Next, we validated the methodology for surgical assistance on 15 participants. A haptic assistance mode was devised based on the proposed PED model to assist inexperienced operators to perform the procedure. The PED model was tested for instrument deviation, penetration force and penetration depth. Preliminary study results showed that participants with PED assistance performed the task with more consistency and exerted lesser penetration force than subjects without assistance.
\end{abstract}

Keywords: Expert demonstration, tele-surgery, robot-assisted, trocar insertion, Gaussian mixture, surgical assistance.

\section{Introduction}

The goal of tele-robotic surgery is to replicate normal surgical processes from a distance. Tele-operated surgical systems have the capability to assist as well as enhance a surgeon's precision and dexterity ${ }^{[1-5]}$. Because of their direct impacts on surgical outcomes, two essential requirements while operating a tele-surgical system are reliability and safety:

Reliability, when the human operator sends commands to the surgical robot, he/she expects instant feedback to reflect the effect of the inputs in a natural and transparent way ${ }^{[3]}$. However, in practice, transmission errors and latencies can exist even when the master controller is located in close proximity to the slave robot. This can render the system unreliable for most surgical applications.

Safety, when the surgeon has difficulty in detecting and reacting to certain situations. These could be unintentional slips, instruments incising out of safe operative margins, or excessive tissue break through force causing rupture of underlying organs. One approach to increasing safety and handling human error is by providing surgical assistance, an ongoing topic of research ${ }^{[6]}$.

In tele-surgery, it is essential that the remote human operator becomes an integral part of the system. Also, the efficacy of tele-robotic surgery depends on how faithfully and without incident the remote robot duplicates its human operators' actions. In this paper, we propose a new predictive methodology to handle both transmission and human errors by characterizing surgical skills. We extend

Manuscript received April 1, 2013; revised August 23, 2013

This work was supported in part by the "Juan de la Cierva" Spanish Program. our prior work on modeling the surgical procedure of trocar insertion ${ }^{[7,8]}$ and sharing of haptics sensations using tele-haptics ${ }^{[9,10]}$ to address the described problems. We apply our approach to tele-robot assisted insertion of the trocar, which is a new application in the field of robotassisted surgery ${ }^{[1-3,11]}$.

Trocar insertion is the first step of any minimally invasive surgeries, where a sharp long tool (called a trocar) is punctured into the pelvic region of the patient (see Fig. 1). This creates the necessary port to insert the laparoscopic and endoscopy tool. This procedure has been extensively cited for causing surgical injuries (most patient injuries appear to occur during initial insertion of the trocar $)^{[12,13]}$. It is performed primarily with only haptic sensation and very little or no visual feedback (while inserting the first trocar). Because of its susceptibility to human error, this procedure is an ideal candidate to validate our proposed methodology.

\subsection{Transmission latency and error back- ground}

Remote surgery requires quick and reliable operation between the surgeon site master and the patient site slave. Researchers have shown that varying latency (due to packet loss and error) significantly reduces the operators' performance both in robotic tele-surgery and in simulated environments ${ }^{[14-17]}$. The general approach to handle latency is to slow down the surgeons' movements, allowing time for the visual feedback to confirm the intended move. However, in some cases, this can prevent critical work by hindering spontaneous reaction to unexpected events. 
Several methods have been proposed for mitigating latency and improving operative reliability. Most common approaches are based on data compression and prediction techniques. Pantel and Wolf ${ }^{[18]}$ proposed a method that uses controller predictions. In [15], the authors used a prediction model that relies on least-square estimation and median filtering to obtain packet reduction rates of $92 \%$. A simple linear prediction method was described in [19], where latency was handled by reducing transmission data. Other approaches to improve reliability by providing realtime network communication were presented in [20,21]. A common approach is to use Kalman filters to compress data $^{[22]}$. In [23], the authors proposed the use of the extended Kalman filter (EKF) to improve reliability in a forceposition application.

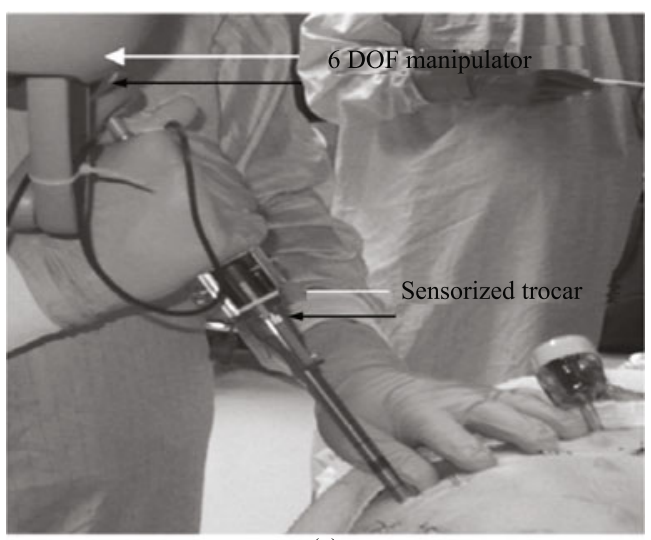

(a)

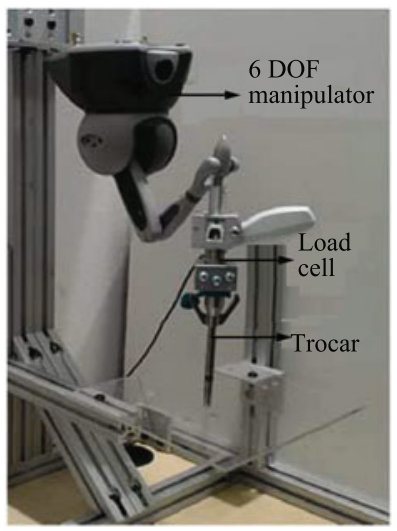

(b)

Fig. 1 Data collection in porcine lab. (a) Data is collected for the procedure of trocar insertion; (b) The sensorized trocar attached to a 6 DOF Phantom Omni

\subsection{Surgical assistance background}

In addition to reliable operation, safety is an essential requirement for any tele-surgical system. Safety requirements can significantly reduce surgical malpractice, for instance, applying safe zones that allow the robot to operate only within the predefined area ${ }^{[24]}$. The ability of a tele-surgical system to provide such assistance leads to a number of promising clinical and scientific opportunities $^{[25]}$. Haptic feedback is generally used to provide the surgeon with an indication when excessive forces are applied. In [26], the authors proposed an algorithm to detect surgical accidents by enhancing force feedback using a bilateral forceps system. The algorithm denotes that the miniaturization of motion and the enhancement of tactile sensations contribute to achieving better dexterity and precision in difficult surgical scenarios. Commonly used constraints such as virtual fixtures ${ }^{[25,27]}$ have been discussed in the context of tele-robotics and surgical robotics. Cha et al. ${ }^{[28]}$ described an approach using depth imagery to generate haptic virtual fixtures. However, the use of virtual fixtures has only limited application in tele-surgery, since such fixtures do not account for variations in patient anatomy, and nonlinear deformation of underlying organs.

\subsection{Our prediction methodology}

We present an alternate approach by moving away from the conventional master slave system, where the slave robot merely mimics the motion of the master. We develop an approach, where the slave robot has some prior information of the procedure. None of the earlier described methods take into consideration prior knowledge (expert model) about the task itself. We turn to the field of intelligent learning and address problems associated with reliability and safety.

Approaches such as fuzzy learning are becoming more and more popular in learning systems ${ }^{[29-31]}$. Liu ${ }^{[30]}$ proposed an aggregation operator to extract robot behaviors for intelligent robotics. Also in [31], the authors proposed a framework for describing articulated robot kinematics in the context of intelligent robotics. Active learning that is based on learning from demonstrations is another method that has received attention ${ }^{[32-34]}$. In the surgical domain, active learning can capture underlying structure of a surgical task (clinical procedure) from training examples ${ }^{[35,36]}$. In this approach, the robot can query its local model whenever it is not confident in a task it is about to perform ${ }^{[32]}$.

In this paper, we propose a new prediction methodology, prediction from expert demonstration (PED), to improve reliability and safety in tele-surgery. We implement this predictive model for tele-robotic placement of a trocar in robot-assisted surgery. In our approach, we make predictions based on demonstrations carried out by an expert. To make predictions from observation (i.e., infer the force, torque and position), data needs to be collected from demonstrations for the task. This data can be exploited and the variability inherent in various demonstrations can lead to extraction of essential components. These essential components are those that remain invariant across the various demonstrations, hence can be used to make reliable predictions.

The remainder of the paper is structured as follows. Data collection for creation of the expert model using ex-vivo demonstrations followed by a new PED model is addressed in Section 2, and Section 2.6 describes the haptic assistance model. Experimental results are presented in Section 3 where we validate the methodology for lost packet and errors, followed by the conclusion in Section 4 . 


\section{Method}

In our proposed PED approach, data (forces / torques and tool trajectories) was collected from expert demonstrations and encoded using a probabilistic model.

\subsection{Data collection}

Expert data was collected in a porcine lab at Roswell Park Cancer Institute (RPCI, Buffalo, NY) and used for generating the predictive model. A non-invasive measuring system was setup to collect data (force, torque and position) for the procedure of trocar insertion (Fig. 1(a)). A force/torque sensor (NANO 25, ATI Industrial Automation, Inc.) mounted at the end effector of a 6 degrees of freedom (DOF) Phantom Omni (Fig. 1 (b) was used to measure force and torque from a $12 \mathrm{~mm}$ pyramidal-blade reusable trocar (Intuitive Surgical, Sunnyvale, CA, USA). We constructed an instrumented trocar of length $340 \mathrm{~mm}$. by attaching the trocar to the base of the force / torque sensor (Fig. 1 (b)). Using this setup, the position of the trocar tip and force/torque values were continuously tracked and recorded. Using the Cartesian coordinates of the trocar tip position from clinical experiments, penetration forces and torques, and the associated force and torque profiles (Fig. 2) were computed. These extracted force and torque profiles are continuous and highly correlated. The profiles were then spatially aligned, resampled and used as the expert data.

\subsection{Task space characterization}

The instrument trajectory (path of the trocar) in the task space is characterized into a set of required operations that are essential to complete the procedure. We consider this trajectory as a set of states (Fig. 2). State A represents the starting position of the trocar, state $\mathrm{B}$ represents the position of trocar tip at the site of entry (port location), and $\mathrm{C}$ represents the final position when penetration of the trocar is complete. In our experimental setup, the abdominal wall thickness is ranged between $18 \mathrm{~mm}$ to $24 \mathrm{~mm}$. The abdominal wall was insufflated to a pressure of $10 \mathrm{mmHg}$. In Figs. 2 (b) and (c), we illustrate the force profile of the trocar insertion procedure as a function of time and penetration depth. The force required to go through the fascia is shown at the first peak from the baseline force measurement. The force required to go through the peritoneum is indicated by the second peak. Once the instrument has penetrated the tissue (Fig. 2 (c)), we observe a drop in the force profile due to loss of resistance after going through the peritoneum. For modeling this task, our goal is to generate a continuous trajectory over all states. Each state profile should connect seamlessly to the other. The states are modeled using a Gaussian mixture model (described next) to characterize the set of constraints (force, torque and penetration depth) that are essential to perform the task. A continuous distribution over all states is obtained using Gaussian mixture regression as described next.

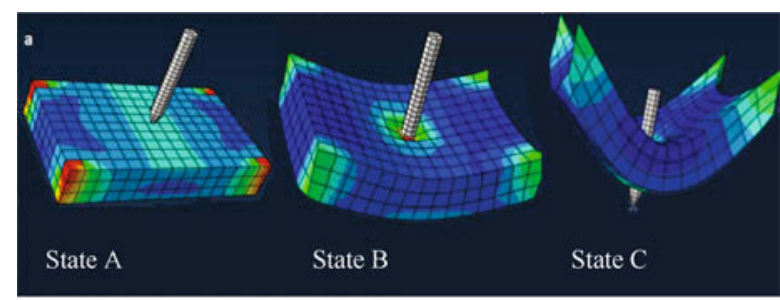

(a)

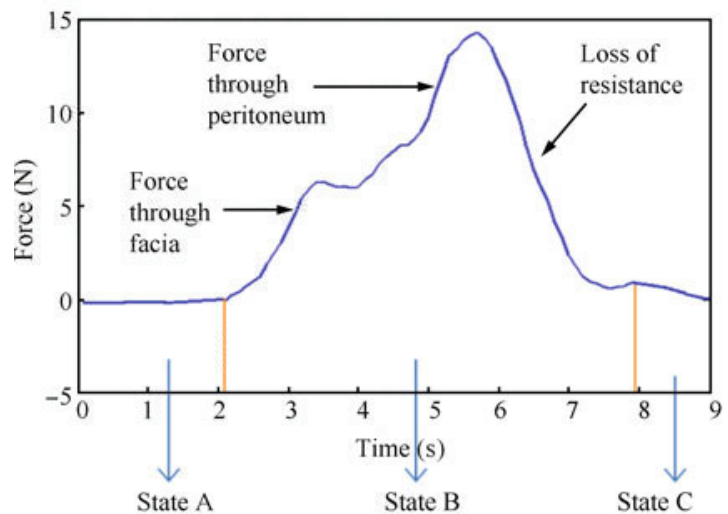

(b)

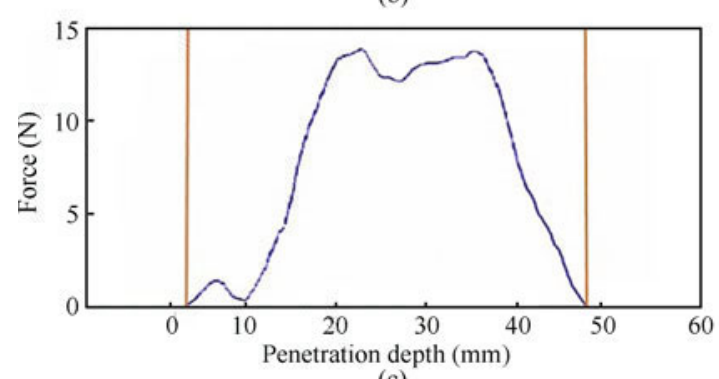

(c)

Fig. 2 Task space characterization showing states of the procedure. (a) Initial state A, where instrument is not in contact with surface. Penetration state B, where instrument is in contact with tissue. Termination state $\mathrm{C}$, where instrument breaks through underlying layer of tissue. (b) and (c) Characteristic force profile of trocar during insertion; they show the instrument forces at various stages of penetration as a function of penetration time and depth respectively

\subsection{Modeling expert demonstrations}

During the data collection phase penetration forces, torques, end effector (trocar tip) positions and deviations are extracted for the set of task trajectories (obtained from clinical experts who were asked to perform this procedure multiple times). Two critical parameters for this procedure are: 1) the force applied by the instrument in contact with tissue (which is dependent on penetration depth), and 2) the maximum penetration of the instrument before breaking through underlying tissue surface. We therefore model the expert data as a function of penetration depth. Profiles of the extracted data are aligned through dynamic warping $(\mathrm{DW})^{[37]}$. Next, we encode this set of expert profiles and retrieve a generalized version of the profile ${ }^{[29,35,36]}$ and associated parameters by using a Gaussian mixture model (GMM), followed by Gaussian mixture regression 
$(\mathrm{GMR})^{[38]}$. A benefit of this approach is that the generalized trajectory is learned through the expectation maximization (EM) algorithm ${ }^{[29,39]}$ which takes into account model variability in a probabilistic framework.

Given $n$ demonstrations of length $m^{i}$, for $i=1 \cdots n$, each demonstration is a vector $y_{\varphi j}^{i}$ composed of forces $f_{j}^{i}$ and $\tau_{j}^{i}$ torques and deviation $x_{j}^{i}$ from mean trajectory for penetration depth $p_{j}^{i}$.

$$
y_{\phi j}=\left[\begin{array}{c}
\phi_{j}^{i} \\
p_{j}^{i}
\end{array}\right], \text { for } j=1, \cdots, m^{i} ; i=1, \cdots, n
$$

where $\varphi=\tau$ for modeling torque, $\varphi=f$ for modeling force, and $\varphi=x$ for modeling deviation from instrument trajectory. Next, the joint density is estimated over the input and output space and is computed as follows.

A GMM of $K$ components is defined by the probability distribution function in (2), where $K$ is determined by the Bayesian information criteria (BIC).

$$
p\left(\varphi_{j}\right)=\sum_{k=1}^{K} p(k) p\left(\varphi_{i} \mid k\right) .
$$

For force and torque predictions, a generalized form of force and torque profiles for a given penetration depth is established (Fig. 3). The same generalization is obtained for deviation from expert trajectory (Fig. 4).

\subsection{Making predictions from expert model}

We can effectively predict the output by conditioning on the joint distribution and taking the expected value. To make predictions, penetration depths are queried for corresponding $\varphi$ estimated through regression. Here, we describe the methodology for force, but the same approach could be generalized for other variables such as deviation and torque. From the GMM the $k$-th Gaussian is given by mean and covariance matrix

$$
\mu_{k}=\left\{\mu_{\varphi k}, \mu_{p k}\right\}, \quad \sum_{k}=\left[\begin{array}{cc}
\sum_{\varphi k} & \sum_{\varphi p k} \\
\sum_{p \varphi k} & \sum_{p k}
\end{array}\right] .
$$

A force (torque and deviation) prediction model is established by GMR. The conditional expectation of given observation $n_{\varphi k}$ and penetration depth are computed as

$$
\hat{n}_{\phi k}=\mu_{\phi k}+\sum_{\phi p k}\left(\sum_{\phi p k}\right)^{-1}\left(n_{p}-\mu_{p k}\right)
$$

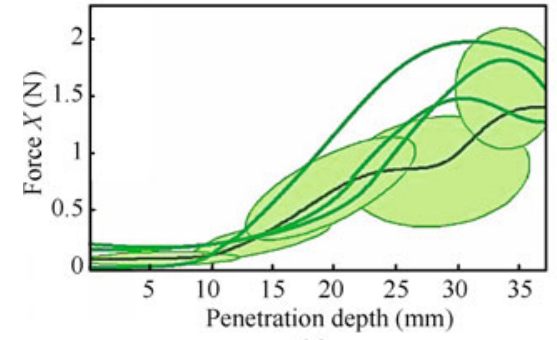

(a)

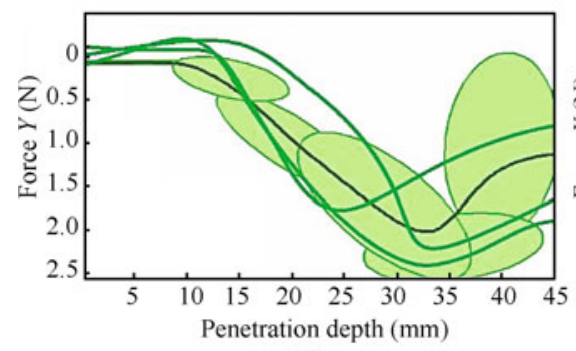

(d)

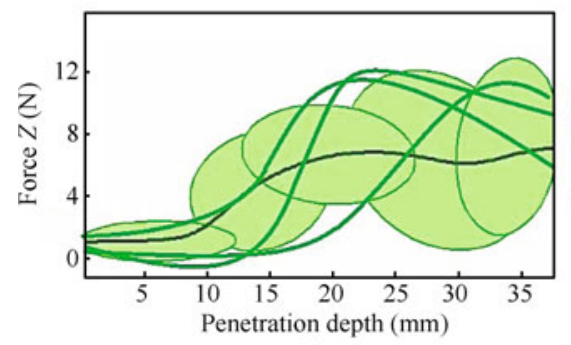

(g)

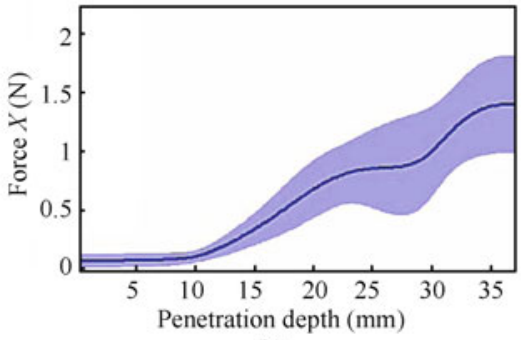

(b)

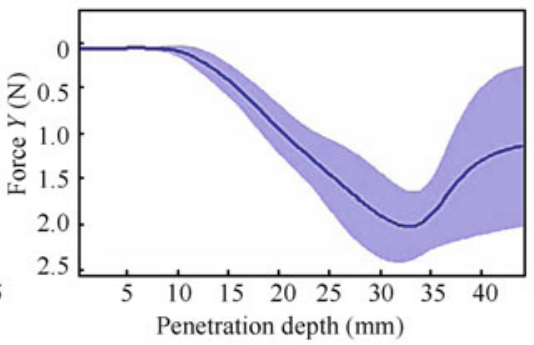

(e)

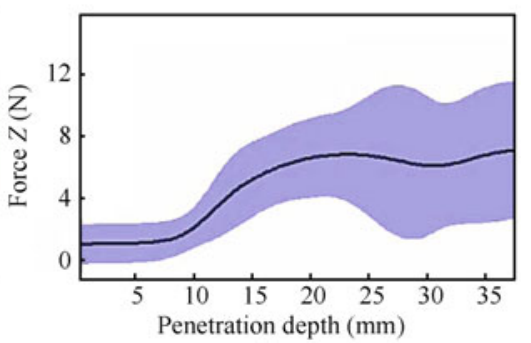

(h)

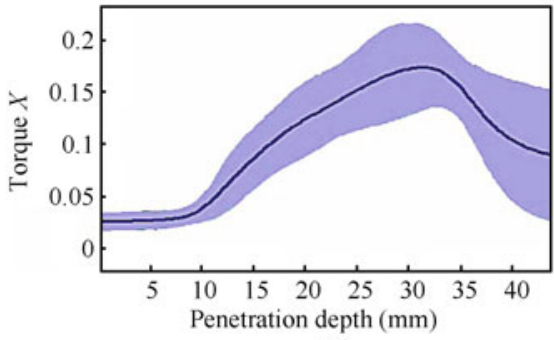

(c)

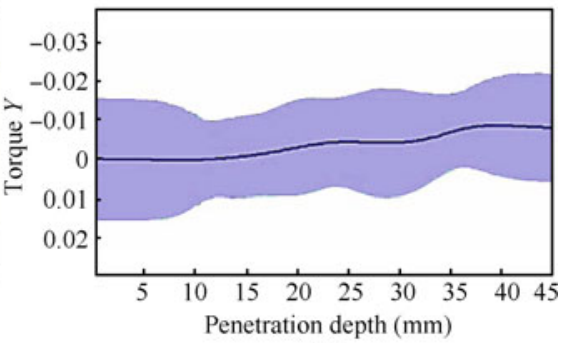

(f)

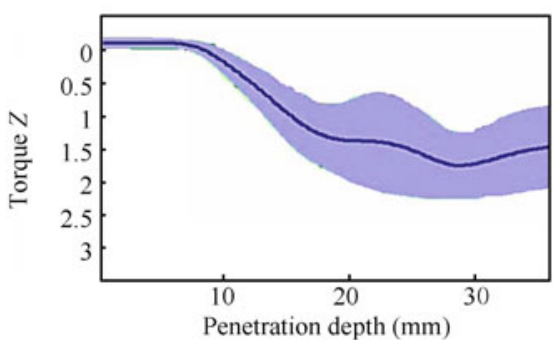

(i)

Fig. 3 Modelling of the force and torque profiles. (a), (d) and (g) GMM of expert force profiles resolved into $x, y, z$, having $k=5$ mixing components; (b), (c), (e), (f), (h) and (i) show the generalization of 1D components of forces and torques using GMR as a function of penetration depth 


$$
\hat{\Sigma}_{\phi k}=\sum_{\phi k}+\sum_{\phi p k}\left(\sum_{p k}\right)^{-1}\left(\sum_{\phi p k}\right)
$$

where the probability of the Gaussian component is given by

$$
\gamma_{K}=\frac{p\left(n_{j} \mid k\right)}{\sum_{i=1}^{k}\left(n_{p} \mid i\right)} .
$$

From (6) we obtain the conditional expectation and covariance given penetration depth

$$
\begin{gathered}
\hat{n}_{\phi}=\sum_{\phi p k}^{K} \gamma(k) \hat{n}_{\phi k} \\
\hat{\Sigma}_{\phi}=\sum_{\phi k}^{K} \gamma(k) \hat{n}_{\phi p k} .
\end{gathered}
$$

By evaluating $\hat{n}_{\phi}$ and $\hat{\Sigma}_{\phi}$ for a given observation of penetration depth, we can generate force, torque and trajectory deviation predictions with given covariance. In Fig. 3, we illustrate the model. The associated GMR representation is depicted where the generalization of the modeled parameters are showed by their Gaussian means. It can be seen that a GMM with 5 mixture components is sufficient to effectively model the instrument force/torque and deviation.
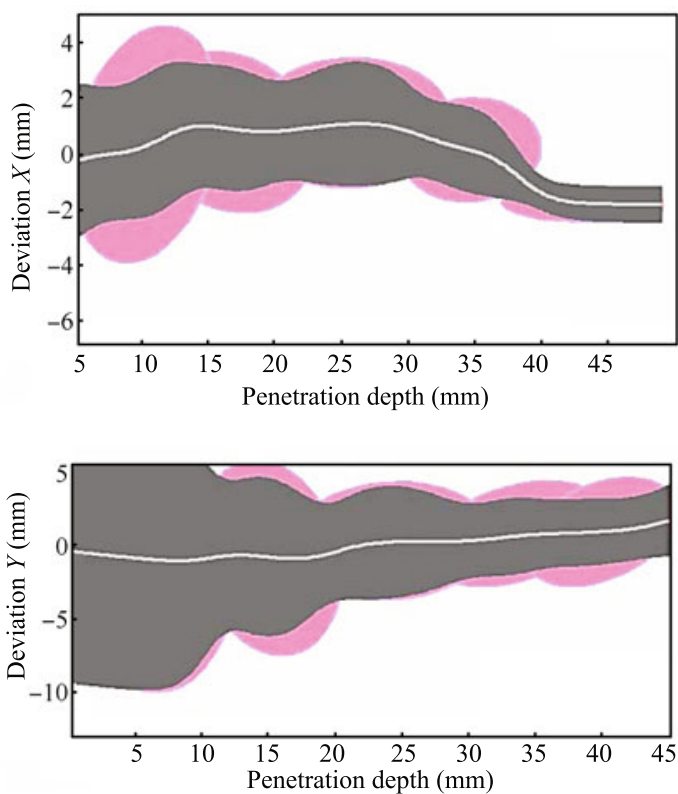

Fig. 4 Generalized lateral deviation along the $X, Y$ axes using GMR form expert trajectory data using $k=5$ mixing components

\subsection{Master and slave mapping}

A mapping is obtained between the haptic master and slave robot (using joint constraints) such that both end effectors attain the same orientation. In our experimental setup, a 6 DOF Phantom 1.5 capable of providing force and torque feedback is served as the master controller (Fig. 5 (a)

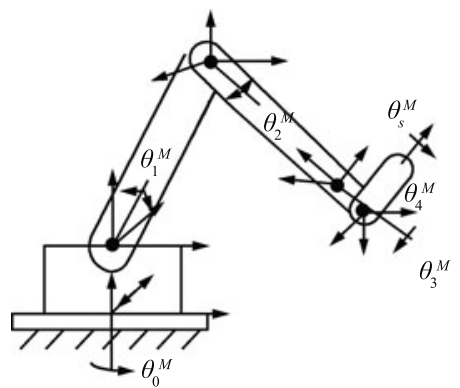

(a)

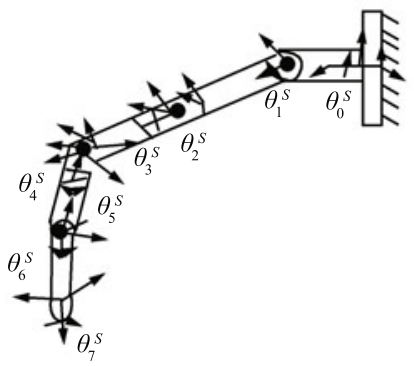

(c)

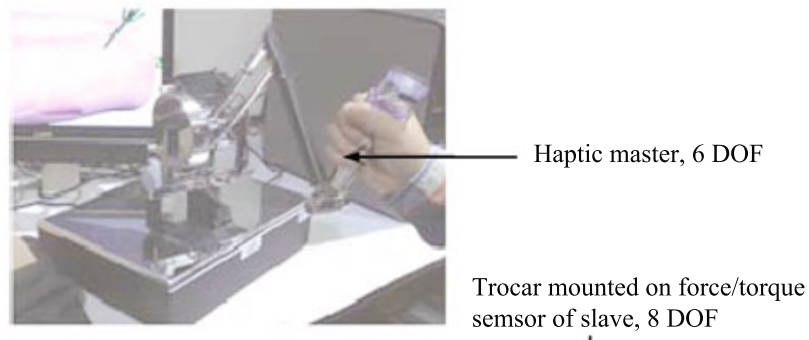

(b)

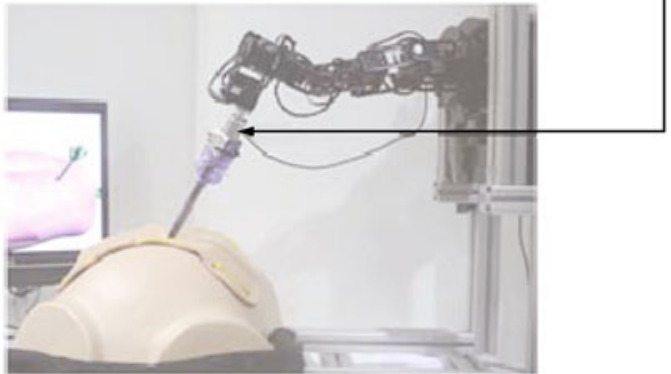

(d)

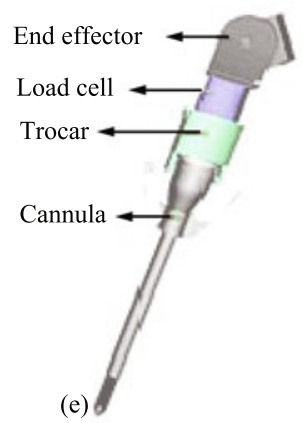

Fig. 5 Joint configurations of the master and slave systems. (a) Joint configuration master haptic system; (b) The Phantom 1.5 master (6 DOF) used as the master controller; (c) Joint configuration of slave robotic system; (d) RobAi slave (8 DOF) with trocar mounted on a force/torque sensor attached to robot end effector (e) 
master and (c) slave). The slave is an 8 DOF robot RobAi with maximum reach of $480 \mathrm{~mm}$. A custom end effector fitted with a detachable trocar mounted on a load cell to monitor end effector force and torque was fabricated (Fig. 5 (c)). The mapping between the master and slave is obtained as follows: The slave can be split into an arm $\left(\theta_{0}^{S} \sim \theta_{3}^{S}\right)$ and a wrist $\left(\theta_{4}^{S} \sim \theta_{7}^{S}\right)$. The arm of the slave consists of a shoul$\operatorname{der}\left(\theta_{0}^{S}, \theta_{1}^{S}\right)$ and an elbow $\left(\theta_{2}^{S}, \theta_{3}^{S}\right)$ with 4 DOF. These 4 DOF of the slave wrist are locked and mapped to the master wrist. The roll of the master wrist $\theta_{5}^{M}$ is mapped to the roll $\theta_{7}^{S}$ of the slave, yaw $n^{\prime \prime} \theta_{4}^{M}$ is mapped to $\theta_{6}^{S}$ and pitch $\theta_{3}^{M}$ is mapped to $\theta_{5}^{S}$ of slave wrist (Figs. $\left.5(\mathrm{~d}),(\mathrm{e})\right)$. In this configuration, the salve attains the same end effector orientation as the master. This mapping is used to set joint constraints for modeling robot kinematics. Since we utilize a redundant manipulator by imposing both joint contrasts and end-effector constraints, the target joint angle configuration is obtained.

For the given manipulator $X_{e}=J_{e}(q) \dot{q}$, where $X_{e}$ is the robot end-effector position and $J_{e}(q)$ is the Jacobian matrix, $q$ is the column of joint angle matrix and $\dot{q}$ the velocity vector, the inverse kinematic solution is given by

$$
\dot{q}=\left(J_{e}^{\dagger}(q)\right) \dot{X}_{e}
$$

where $J_{e}^{\dagger}$ is the Moore-Penrose pseudo-inverse of the endeffector Jacobian matrix. We compute $\dot{q}^{\prime}$ such that reconstruction error $X_{c}-J_{c} q_{0}$ is minimized, where $\dot{X}_{c}$ is constraint task velocity vector, $J_{c}=\partial X_{c} / \partial q$ and $q_{0}$ are arbitrary joint velocity vectors.

$$
\dot{q}=J_{e}^{\dagger} \dot{X}_{e}+\left(J_{c}\left(I-J_{e}^{\dagger} J_{e}\right)\right)^{\dagger}\left(\dot{X}_{c}-J_{c} J_{e}^{\dagger} \dot{X}_{e}\right)
$$

where $I$ is the identity matrix. The damped least squares inverse of Jacobian is given by

$$
J_{e}^{\dagger}=J_{e}^{\mathrm{T}}\left(J_{e} J_{e}^{\mathrm{T}}+\lambda^{2} I\right)^{-1}
$$

where $\lambda$ is the damping factor. The execution of endeffector motion is performed as follows. The error $e_{e}$ has the position and orientation given by

$$
e_{e}=\left[\begin{array}{c}
P_{d}-P \\
\frac{1}{2}\left(n \times n_{d}+s \times s_{d}+a \times a_{d}\right)
\end{array}\right]
$$

where $P_{d}$ and $P$ are the $3 \times 1$ vectors of the desired and actual position of the end-effector and $R_{d}=\left(n_{d} s_{d} a_{d}\right)$ and $R=(n s a)$ are the $3 \times 3$ rotation matrices representing the desired and actual orientations. For a detailed description of this approach, we refer the reader to $[40,41]$.

\subsection{Haptic assistance model}

A force-reflecting robot assistance scheme was implemented to communicate position control commands to the slave and provide haptic feedback to the master. This provides sensory input in the form of haptic feedback to the operator, and allows the operator to judge the nature of the force and take necessary action. This approach draws on prior work performed on shared control and virtual fixtures $^{[42]}$.

Haptic assistance is provided as force reflection (haptic feedback) at the master controller when the PED model constraints are exceeded. In our approach, stiffness is induced as motor (joint) torques given by a simple mass and damper system $F=K x+b x^{\prime}$. When the master is outside the region predicted by the model, these motor torques prevent the master from deviating from the PED model. The haptic feedback provided is proportional to the deviation of the master from the model trajectory given by

$$
P_{n}=\left(p_{n}-p_{s}\right)
$$

where $P_{n}$ is the difference between the maximum estimated model prediction $p_{n}\left\{p_{x n}, p_{y n}, p_{z n}\right\}$ position (force/torque), and $p_{s}\left\{p_{x s}, p_{y s}, p_{z s}\right\}$ is the position (force/torque) of the slave. Force reflection (assistive force and torque feedback) on the master is given by

$$
\begin{aligned}
& F_{m}=F_{s}+K\left(P_{n}\right)+b V \\
& \tau_{m}=\tau_{s}+K\left(P_{n}\right)+b V
\end{aligned}
$$

where $F_{s}\left\{F_{x s}, F_{y s}, F_{z s}\right\}$ is the force, and $\tau_{s}\left\{\tau_{x s}, \tau_{y s}, \tau_{z s}\right\}$ is the torque sensed by the slave robot. $F_{m}$ is the force feedback and $\tau_{m}$ is the torque feedback at the master, $b$ is the damping constant, and $V$ is the velocity of the master controller. In our experimental setup, the stiffness $K$ was set at $0.5 \mathrm{~N} / \mathrm{mm}$ so that the maximum haptic stiffness could be sensed (during trocar penetration of the surrogate phantom model, Fig. 5 (b))

\subsection{Phantom setup}

In Fig. 5, we illustrate our surrogate phantom setup. A Phantom 1.5 haptic device was fitted with a custom end effector and used as the haptic master. The slave was an 8 DOF RobAi fitted with a sensorized trocar mounted on the end effector (Fig. 5). A human abdominal phantom with surrogate abdominal tissue (on the lower abdominal section) having similar viscoelastic properties to that of human abdominal tissue was used to test for tele-robotic trocar penetration. We used polyethylene to simulate the skin and fat layer and reinforcing nylon tape to simulate the tough muscle layer. The synthetic tissue was tested in both wetted conditions using physiologic saline solution, and in dry conditions. The penetration force profiles obtained using this synthetic tissue were similar to that shown in Fig. 2. Similar materials have been used in prior research by Baier ${ }^{[43]}$ and have been validated against human tissue properties as an excellent yet cheap alternative for human skin tissues.

The surrogate tissue was stretched and clamped over an inanimate abdominal phantom using metal clamps. In our experiments the trocar attached to the end effector of the tele-controlled slave was advanced by the master. The force and displacement of the trocar were recorded by load cell of the slave and reflected on the master as haptic force feedback. 


\subsection{Tele-operation network model}

The master slave network model was implemented as follows. Both master and slave tele-communicate through PED used user datagram protocol (UDP) packets. As shown in Fig. 6, the master and slave devices are connected through a $100 \mathrm{Mb} / \mathrm{s}$ local network. This congestion type was selected because UDP is a non-TCP-friendly protocol, therein allowing the simulation of a more aggressive networking environment typically used by internet programs like P2P.

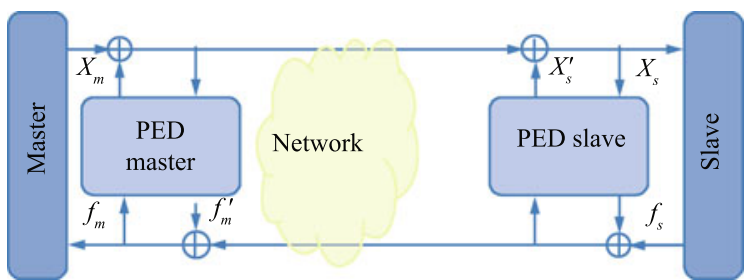

Fig. 6 Operation of the tele-robot system using PED through $100 \mathrm{Mb} / \mathrm{s}$ local network, where $X_{m}$ and $X_{s}$ are the position, and $f_{m}$ and $f_{s}$ are the force and torque of the master and slave, respectively

The advantage of using UDP in tele-operation is that communications can be carried out without considerable delays or fluctuations (jitter) when network conditions are favorable (without congestion). On the other hand, when congestion problems appear in the network, UDP cannot guarantee reliable data transmission for robotics teleoperation. Also, UDP provides very few error recovery services, offering instead a direct way to send and receive datagrams. Due to these limitations, other control techniques ${ }^{[4,45]}$ are required to guarantee optimal tele-operation. We establish a bi-directional transmission model ${ }^{[4]}$ that operates through PED for master and slave communication. This is an end-to-end congestion control protocol whose main objective is to minimize the round trip time (RTT) while maximizing the transmission frequency. It performs network congestion control by means of avoiding congestion signals (timeouts and packet losses) and handles instability due to phase and transmission delays. We refer the reader to our prior work ${ }^{[44]}$ for a more detailed description on this transmission protocol.

In our experiments, additional UDP flows were included as network traffic in order to simulate network congestions. At the slave end PED, acts as a predictor providing for error correction in the presence of lost packets or human error. On the master side, PED (Fig. 6) provides for surgical assistance by inducing a haptic force feedback. The communication frequency between master and slave was set to $1 \mathrm{kHz}$. Theoretically, this would mean that one packet of data was received per millisecond. However, factors such as delay, jitter and packet loss would alter this rate. The effects of this are: Loss packets will decrease the slave frequency, delayed packets will cause position, force/torque errors, and jitter (due to packets arriving out of time sequence) will cause instability in the system. We address these issues using the PED model where both master and slave can operate at the same frequency.

\section{$3 \quad$ Experiment and results}

We tested our proposed methodology for tele-robotic placement of the trocar. For the surgical task of trocar insertion, our PED model has to compensate for kinematic differences and latencies between the master controller and the slave robot in the presence of transmission and human error. We validated the model based on two criteria: 1) recovery from transmission errors and data loss, and 2) surgical assistance when model constraints are exceeded due to operator error. For the first case we test for reliable operation of the system in the presence of transmission error. Comparisons are made between predictions made by the extended Kalman filter (EFK) and the PED model. For the second case, we tested for safety, the ability of the model to provide haptic assistance to prevent and handle human operator error.

\subsection{Experimental study 1: Transmission errors and packet loss}

In this part of the experimental study, our proposed PED method is evaluated for latency and error. Experiments were performed in the context of learning from demonstration strategy for error recovery.

Master end effector positions are transmitted to the slave for tele-control, and force/torque data at the slave end is transmitted to the master for haptic reflection (of trocar penetration forces). We evaluate the effectiveness of the model in the presence of transmission error. Comparisons are made between the proposed PED model and a filtering approach that utilizes an extended Kalman filter ${ }^{[23]}$. We test for transmission error in the ranges $1 \%, 12 \%, 25 \%$ and $50 \%$ and data loss in the ranges $5 \%, 10 \%, 20 \%$ and $30 \%$ (Fig. 7). The efficacies of the two approaches are analyzed by comparing their root mean square (RMS) error between transmitted and received data.

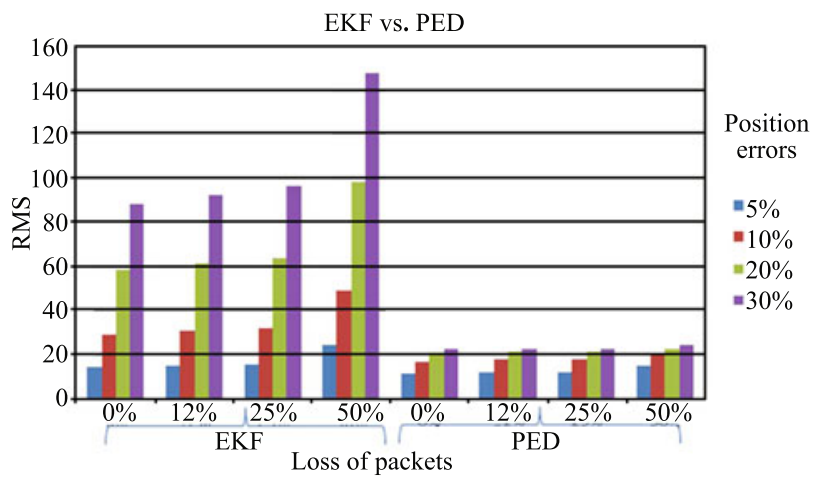

Fig. 7 The root mean square (RMS) position error between EKF and PED. Different bars indicate percentage of lost packet and the $x$-axis gives the transmission error

Fig. 7 gives our results for transmission error of end effector positions. The horizontal axis indicates the percentage of transmission errors, and the different bars give the percentage of data packets lost at the receiver. It can be 
observed that the RMS error using only the EKF is greater than the RMS error obtained using the PED model. When packet loss is 5\%, both the PED and EKF have comparable performance, over increasing error rates (EKF error ranges form 14.6-24.5 and PED error ranges from 11.315). The benefit of using the PED model can be seen in situations of packet loss between $20 \%$ to $30 \%$. We observe that the error in the EKF increases ten folds with increase in transmission loss (EKF error ranges from 14.6-147.5). This is expected since the EKF makes predictions on the data using the covariance in prior observations and not on how the data is generated (such as an expert model). The PED shows significantly better performance with RMS error ranging from 11.3-24.1 (Fig. 8). In the average case (12\% error and $10 \%$ loss) we obtain a $58.8 \%$ improvement in performance in comparison to using an EKF. The PED having prior knowledge of the underlying model that generated the data is capable of making better predictions even when there is significant loss in packets.

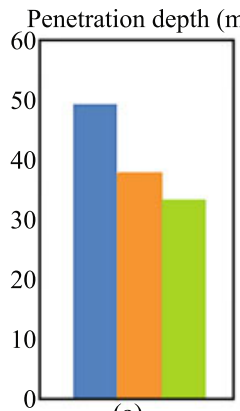

(a)

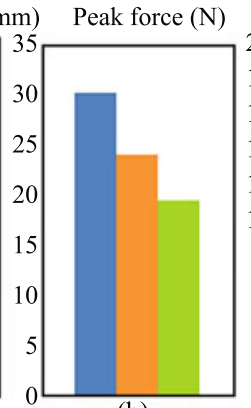

(b)

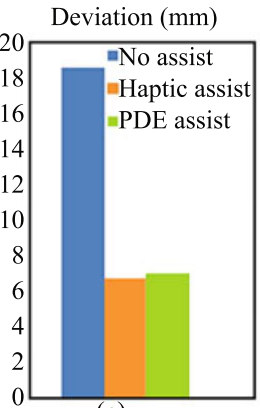

(c)
Fig. 8 Plots show (a) mean penetration depth (PD), (b) instrument deviation (DV) and (c) peak force (PF). Group A is provided with PED assistance (PEDA), Group B is provided with only haptic assistance (HA) and Group $\mathrm{C}$ is provided with no assistance (NA)

Equally important to transmission of position is the transmission of force between the slave and master for haptic feedback (requiring higher transmission rates). Maximum penetration forces (in Newtons) ranged from $59.6 \mathrm{~N}-73.1 \mathrm{~N}$. Comparisons are made between the performance of the EKF and the PED model for force ranges: $0.5 \mathrm{~N}, 1 \mathrm{~N}, 1.5 \mathrm{~N}$ and $2 \mathrm{~N}$. The RMS error in the case of EKF (for increasing transmission loss, 5\%-30\%) varied from 2.27-10.3. Significantly better performance was obtained using PED with RMS error ranging from 1.96 to 5.08. Here the error using EKF was twice that of PED. In the average case ( $12 \%$ error and $10 \%$ loss), we obtain a $42.6 \%$ improvement in performance in comparison to using PED than EKF.

\subsection{Experimental study 2: Surgical assis- tance}

The proposed PED methodology was validated for haptic assistance and usability in the following scenarios: 1) Penetration depth assistance, when the operator attempts to penetrate below the expert model (PED) depth. 2) Task trajectory deviation, when the operator deviates beyond the predicted deviation of the model. 3) Force/torque assistance, when the operator applies penetration forces and torques beyond what the model predicts. From prior work $^{[7,8]}$, the mean penetration depth of the trocar through the abdominal wall was estimated to be approximately $45 \mathrm{~mm}$. In our experimental setup, this was set as the maximum penetration depth. Force reflection of the slave at the master end enables the operator to sense penetration forces/torque of the slave. Surgical assistance was provided at the master by a haptic feedback (joint stiffness) that was proportional to the deviation of the slave from model predictions.

For validation, novices who had no prior experience in performing trocar insertion procedure were selected. Data was collected from 15 participants, formed into three randomized groups $\mathrm{A}, \mathrm{B}$ and $\mathrm{C}$ (with 5 participants each). Group A (no assistance), as evident by the name, had no haptic feedback and participants had to rely solely on visual feedback for trocar penetration. Group B (haptic assistance) had both visual and haptic feedbacks (force reflection), and Group C (PED assistance) had visual and PED haptic feedbacks (see Tables 1 and 2). Each participant was required to perform 5 trials of the trocar insertion procedure on the phantom setup (Section 2.7). The participants manipulated the trocar using the haptic master controller (Fig. $5($ a)).

Table 1 Groups A, B and C, overall comparisons of mean penetration depth $(\mathrm{PD})$, mean peak force $(\mathrm{PF})$, mean instrument deviation (DV), $p$-values shows significance

\begin{tabular}{lcccc}
\hline & $\begin{array}{c}\text { Group A } \\
(\mathrm{NA})\end{array}$ & $\begin{array}{c}\text { Group B } \\
(\mathrm{HA})\end{array}$ & $\begin{array}{c}\text { Group C } \\
(\text { PEDA })\end{array}$ & $p$-value \\
& $(\mathrm{mm})$ & $(\mathrm{mm})$ & $(\mathrm{mm})$ & \\
\hline Mean PD & 49.44 & 37.94 & 33.44 & 0.0061 \\
Mean PF & 30.24 & 24.02 & 19.44 & 0.0073 \\
Mean DV & 18.5 & 6.72 & 7.02 & 0.0024 \\
\hline
\end{tabular}

Table 2 Exact Kruskal-Wallis tests for between group comparison; Haptic assistance (HA), PED assistance (PEDA), No assistance (NA), for penetration depth (PD), peak force $(\mathrm{PF})$ and instrument deviation (DV)

\begin{tabular}{cccc}
\hline & $\mathrm{PD}(p$-value $)$ & $\mathrm{PF}(p$-value $)$ & $\mathrm{DV}(p$-value $)$ \\
\hline HA vs. NA & 0.0516 & 0.0581 & 0.0062 \\
PEDA vs. NA & 0.0041 & 0.0043 & 0.0079 \\
PEDA vs. HA & 0.2344 & 0.2222 & 0.8413 \\
\hline
\end{tabular}

Statistical analysis: Exact Kruskal-Wallis tests were utilized to statistically compare outcomes across independent groups (Group A, Group B and Group C). Between group comparisons (Table 2) showed $\mathrm{C}$ has significantly better outcomes compared to Group A (at 0.05 nominal significance level), with $p$-value of 0.0041 for penetration depth (PD), $p$-value of 0.0043 for peak force (PF) and $p$-value of 0.0079 for instrument deviation (DV). Fig. 6 (a) shows the results for the three groups. Group $\mathrm{C}$ had the least mean 
PD of $33.44 \mathrm{~mm}$. Whereas Group A had mean PD $49.2 \mathrm{~mm}$, exceeding the maximum permissible depth of $45 \mathrm{~mm}$. Similar results were observed for instrument deviation, both Groups B and C performed significantly better with mean deviation of $7.02 \mathrm{~mm}$ and $6.72 \mathrm{~mm}$, respectively, whereas A had mean DV of $18.5 \mathrm{~mm}$. Also for peak force, Group $\mathrm{C}$ exerted less force, completing the task with mean PF of 19.44 N. Whereas, A had a mean PF of 30.24 N. In all cases, our results showed participants with both PED and haptic assistance performed the task better and with more consistency (not exceeding maximum penetration depth) and lesser deviation (closer to the expert model) than subjects without assistance.

Usability of the proposed system was measured by participants' (all groups) ability to complete the task of trocar insertion. Our results demonstrated that all participants were able to successfully complete the task (Table 1 ).

\section{Discussion and conclusions}

In this paper, we propose a new methodology based on prediction from expert demonstrations (PED) for safe telesurgery. Our methodology is tested for tele-robotic placement of the trocar. The quantitative results above validate the efficacy of the proposed methodology for reliability and safety. We show that our PED approach is reliable, robust to transmission error, and provides optimal results in all tested cases. The PED produced significantly better performance in comparison to the EKF, in the average case (12\% error and $10 \%$ loss), and obtained a $58.8 \%$ improvement in performance compared to using the EKF. This robustness is mainly attributable to the probabilistic learning approach of the model. Next, we validate the methodology for safety on a randomized control study of 15 subjects. The main purpose of this experimental study was to demonstrate the effectiveness of the methodology for surgical assistance. Preliminary study results showed participants with PED assistance performed the task with more consistency, closer to the expert model than subjects without assistance.

As demonstrated, our initial study findings validate the effectiveness of the proposed method. Such approach can prove to be beneficial especially to the domain of surgical robotics, where both human operation and data transmission play a critical role. Future studies will utilize a larger study group that include experts to test this approach. We also plan on extending this approach to surgical procedures that involve complex trajectories, and the application of precise forces.

\section{Acknowledgments}

The authors would like to thank Dr. T. Kesavadas for his encouragement and support.

\section{References}

[1] A. Hassan-Zahraee, B. Herman, J. Szewczyk. Mechatronic design of a hand-held instrument with active trocar for laparoscopy. In Proceedings of 2011 IEEE International
Conference on Robotics and Automation, IEEE, Shanghai, China, pp. 1890-1895, 2011.

[2] P. Queirós, R. Cortesao, C. Sousa. Haptic tele-manipulation for robotic-assisted minimally invasive surgery with explicit posture control. In Proceedings of the 18th Mediterranean Conference on Control \& Automation (MED), IEEE, Marrakech, Morocco, pp. 808-814, 2010.

[3] T. Haidegger, B. Zoltán. Extreme telesurgery. Robot Surgery, S. H. Baik, Ed., Croatia: InTeck, pp. 25-44, 2010.

[4] J. Ding, R. E. Goldman, K. Xu, P. K. Allen, D. L. Fowler, N. Simaan. Design and coordination kinematics of an insertable robotic effectors platform for single-port access surgery. IEEE/ASME Transactions on Mechatronics, vol. 18, no. 5, pp. 1612-1624, 2013.

[5] J. Wall, J. Marescaux. History of telesurgery. Telemicrosurgery, France: Springer, pp. 15-18, 2013.

[6] A. M. Okamura, L. N. Verner, T. Yamamoto, J. C. Gwilliam, P. G. Griffiths. Force feedback and sensory substitution for robot-assisted surgery. Surgical Robotics: Systems Applications and Visions, New York: Springer, pp. 419-448, 2011.

[7] V. Arulesan, G. Srimathveeravalli, T. Kesavadas, P. Nagathan, R. E. Baier. Data acquisition and development of a trocar insertion simulator using synthetic tissue models. Studies in Health Technology and Informatics, vol. 125, pp. 25-27, 2007.

[8] T. Kesavadas, G. Srimathveeravalli, V. Arulesan. Parametric modeling and simulation of trocar insertion procedure. Studies in Health Technology and Informatics, vol.119, pp. 252-254, 2006.

[9] D. Joshi, T. Kesavadas. Framework for network based manufacturing training through telehaptics. In Proceedings of ASME 2003 International Mechanical Engineering Congress and Exposition, ASME, Washington, DC, USA, pp.707$712,2003$.

[10] A. Chowriappa, R. Wirz, Y. W. Seo, A. Reddy, T. Kesavadas, P. Scott, K. Guru, T. Kesavadas. A predictive model for haptic assistance in robot assisted trocar insertion. In Proceedings of IEEE World Haptics Conference (WHC), IEEE, Daejeon, Korea, pp. 121-126, 2013.

[11] J. Ding, R. E. Goldman, K. Xu, P. K. Allen, D. L. Fowler, N. Simaan. Design and coordination kinematics of an insertable robotic effectors platform for single-port access surgery. IEEE/ASME Transactions on Mechatronics, vol. 18, no. 5, pp. 1612-1624, 2013.

[12] S. Krishnakumar, P. Tambe. Entry complications in laparoscopic surgery. Journal of Gynecological Endoscopy and Surgery, vol. 1, no. 1, pp. 4-11, 2009.

[13] R. Orlando, P. Palatini, F. Lirussi. Needle and trocar injuries in diagnostic laparoscopy under local anesthesia: What is the true incidence of these complications? Journal of Laparoendoscopic \& Advanced Surgical Techniques, vol. 13 , no. 3 , pp. 181-184, 2003. 
[14] J. Y. Chen, E. C. Haas, M. J. Barnes. Human performance issues and user interface design for teleoperated robots. IEEE Transactions on Systems, Man and Cybernetics, Part C: Applications and Reviews, vol.37, no. 6, pp. 1231-1245, 2007.

[15] P. Quax, P. Monsieurs, W. Lamotte, D. De Vleeschauwer, N. Degrande. Objective and subjective evaluation of the influence of small amounts of delay and jitter on a recent first person shooter game. In Proceedings of the 3rd ACM SIGCOMM Workshop on Network and System Support for Games, ACM, New York, NY, USA, pp. 152-156, 2004.

[16] J. Marescaux, J. Leroy, F. Rubino, M. Smith, M. Vix, M. Simone, D. Mutter. Transcontinental robot-assisted remote telesurgery: Feasibility and potential applications. Annals of Surgery, vol. 235, no. 4, pp. 487-492, 2002.

[17] M. Anvari, T. Broderick, H. Stein, T. Chapman, M. Ghodoussi, D. W. Birch, C. Mckinley, P. Trudeau, S. Dutta, C. H. Goldsmith. The impact of latency on surgical precision and task completion during robotic-assisted remote telepresence surgery. Computer Aided Surgery, vol. 10, no. 2, pp. 93-99, 2005.

[18] L. Pantel, L. C. Wolf. On the suitability of dead reckoning schemes for games. In Proceedings of the 1st Workshop on Network and System Support for Games, ACM Press, New York, NY, USA, pp. 79-84, 2002.

[19] Y. Yonghee, Y. S. Mee. Haptic data transmission based on the prediction and compression. In Proceedings of IEEE International Conference on Communications, IEEE, Beijing, China, pp. 1824-1828, 2008.

[20] P. Hinterseer, S. Hirche, S. Chaudhuri, E. Steinbach, M. Buss. Perception-based data reduction and transmission of haptic data in telepresence and teleaction systems. IEEE Transactions on Signal Processing, vol. 56, no. 2, pp. 588597, 2008.

[21] S. Hirche, M. Buss. Transparent data reduction in networked telepresence and teleaction systems. Part II: Timedelayed communication. Presence: Teleoperators and Virtual Environments, vol. 16, no. 5, pp. 532-542, 2007.

[22] R. Hinterseer, E. Steinbach, S. Chaudhuri. Perceptionbased compression of haptic data streams using Kalman filters. In Proceedings of IEEE International Conference on Acoustics, Speech and Signal Processing, IEEE, Toulouse, France, vol. 5, pp. 473-476, 2006.

[23] W. Zarrad, P. Poignet, R. Cortesao, O. Company. Stability and transparency analysis of a haptic feedback controller for medical applications. In Proceedings of the 46th IEEE Conference on Decision and Control, IEEE, New Orleans, LA, USA, pp. 5767-5772, 2007.

[24] J. H. Cho, H. I. Son, M. Annerstedt, A. Robertsson, R. Johansson. Enhancement of human operator's perceptual sensitivity for telesurgical systems via polytopic system approach. In Proceedings of the 4th IEEE RAS \& EMBS International Conference on Biomedical Robotics and Biomechatronics (BioRob), IEEE, Rome, pp. 75-80, 2012.
[25] J. J. Abbott, P. Marayong, A. M. Okamura. Haptic virtual fixtures for robot-assisted manipulation. Robotics Research, Berlin Heidelberg: Springer, vol. 28, pp. 49-64, 2007.

[26] A. M. Harsha, S. Abeykoon, K. Ohnishi. Tactile sensation improvement of a bilateral forceps robot with a switching virtual model. In Proceedings of the 10th IEEE International Workshop on Advanced Motion Control, IEEE, Trento, Italy, pp. 526-531, 2008.

[27] L. B. Rosenberg. Virtual fixtures: Perceptual tools for telerobotic manipulation. In Proceedings of IEEE Virtual Reality Annual International Symposium, IEEE, Seattle, WA, USA, pp. 76-82, 1993.

[28] J. Cha, S. M. Kim, I. Oakley, J. Ryu, K. H. Lee. Haptic interaction with depth video media. Advances in Multimedia Information Processing-PCM, Berlin Heidelberg: Springer, pp. 420-430, 2005.

[29] Z. J. Ju, H. H. Liu. Fuzzy Gaussian mixture models. Pattern Recognition, vol. 45, no. 3, pp. 1146-1158, 2012.

[30] H. H. Liu. A fuzzy qualitative framework for connecting robot qualitative and quantitative representations. IEEE Transactions on Fuzzy Systems, vol.16, no.6, pp. 15221530, 2008.

[31] H. H. Liu, D. J. Brown, G. M. Coghill. Fuzzy qualitative robot kinematics. IEEE Transactions on Fuzzy Systems, vol. 16, no. 3, pp. 808-822, 2008.

[32] S. Chernova, M. Veloso. Confidence-based policy learning from demonstration using Gaussian mixture models. In Proceedings of the 6th International Joint Conference on $\mathrm{Au}-$ tonomous Agents and Multiagent Systems, ACM New York, NY, USA, Article No. 233, 2007.

[33] D. H. Grollman, O. C. Jenkins. Dogged learning for robots. In Proceedings of IEEE International Conference on Robotics and Automation, IEEE, Roma, pp. 2483-2488, 2007.

[34] A. P. Shon, D. Verma, R. P. N. Rao. Active imitation learning. In Proceedings of the 22nd National Conference on Artificial Intelligence, AAAI, Vancouver, Canada, pp. 756$762,2007$.

[35] A. Coates, P. Abbeel, A. Y. Ng. Learning for control from multiple demonstrations. In Proceedings of the 25th International Conference on Machine Learning, ACM, New York, USA, pp. 144-151, 2008.

[36] A. Billard, S. Calinon, R. Dillmann, S. Schaal. Robot programming by demonstration. Handbook of Robotics, New York: Springer, pp. 1371-1394, 2008.

[37] H. Sakoe, S. Chiba. Dynamic programming algorithm optimization for spoken word recognition. IEEE Transactions on Acoustics, Speech and Signal Processing, vol. 26, no. 1, pp. 43-49, 1978 .

[38] S. Calinon, A. Billard. A probabilistic programming by demonstration framework handling constraints in joint space and task space. In Proceedings of the IEEE/RSJ International Conference on Intelligent Robots and Systems, IEEE, Nice, pp. 367-372, 2008. 
[39] A. P. Dempster, N. M. Laird, D. B. Rubin. Maximum likelihood from incomplete data via the em algorithm. Journal of the Royal Statistical Society, vol. 39, no. 1, pp. 1-38, 1977.

[40] Y. Nakamura, H. Hanafusa. Inverse kinematic solutions with singularity robustness for robot manipulator control ASME Journal of Dynamic Systems, Measurement, and Control, vol. 108, no. 3, pp. 163-171, 1986

[41] G. Schwarz. Estimating the dimension of a model. The Annals of Statistics, vol. 6, no. 2, pp. 461-464, 1978.

[42] G. Srimathveeravalli, V. Gourishankar, A. Kumar, T. Kesavadas. Experimental evaluation of shared control for rehabilitation of fine motor skills. ASME Journal of Computing and Information Science in Engineering, vol. 9, no. 1, 014503, 2009

[43] R. E. Baier. Cutting Effectiveness of Heel Incision Devices. Internal UB Technical Report, 2006.

[44] R. Wirz, R. Marin, M. Ferre, J. Barrio, J. M. Claver, J. Ortego. Bidirectional transport protocol for teleoperated robots. IEEE Transactions on Industrial Electronics, vol. 56, no. 9, pp. 3772-3781, 2009.

[45] T. Slama, A. Trevisani, D. Aubry, R. Oboe, F. Kratz. Experimental analysis of an internet-based bilateral teleoperation system with motion and force scaling using a model predictive controller. IEEE Transactions on Industrial Electronics, vol. 55, no. 9, pp. 3290-3299, 2008.

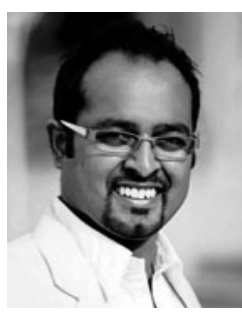

Ashirwad Chowriappa received his $\mathrm{Ph}$. D. degree in computer vision and artificial intelligence from the State University of New York, Buffalo, USA in 2013. He now holds a research associate position at Roswell Park Cancer Institute, Buffalo, New York, USA. He worked as senior research engineer at Simulated Surgical Systems LLC from 2010 to 2012, where his research was focused on both simulation and augmented reality procedure-based training for robot-assisted surgery (RAS). During his educational career, he has authored or co-authored over 30 technical papers in top conferences and journals. He was invited speaker at the Robotic Assisted Microsurgical and Endoscopic Society, Tampa, 2012. He has been a reviewer for IEEE World Haptics Conference, Intelligent Robots and Systems, Systems, Man, and Cybernetics, and ComputerAided Design Journal.

His research interests include computer vision for robotassisted surgery, computer aided diagnosis, and augmented reality in simulation-based robotic training.

E-mail: ajc48@buffalo.edu (Corresponding author)

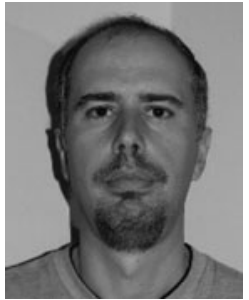

Raul Wirz received his $\mathrm{Ph} . \mathrm{D}$. degree in computer science and engineer form the University of Castellon, Spain in 2009. He completed his post-doctorate in the Centre of Automatic and Robotic (CAR-UPM, Spain) and in the State University of New York at Buffalo, USA in 2011.

His research interests focuses on human robot interfaces and haptic devices in real and virtual environments.

E-mail: raul.wirz@gmail.com

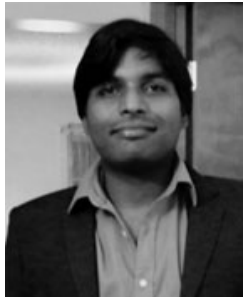

Aditya Reddy Ashammagari graduated from the State University of New York at Buffalo (UB), USA in 2013. He received his M. Sc. degree in mechanical and aerospace engineering from UB in 2013 and B. Sc. degree in mechatronics engineering from SASTRA University, Tanjore, India in 2011.

His research interests include robotics and automation, vision based control, machine intelligence, computer vision, virtual reality and graphics. E-mail: aditya.ash@gmail.com

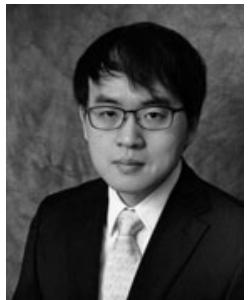

Yong Won Seo is a $\mathrm{Ph} . \mathrm{D}$. candidate in mechanical and aerospace engineering at the University at Buffalo, USA. He received his M.Sc. degree in mechatronics from Gwangiu Institute of Science and Technology (GIST), South Korea, and B. Sc. degree in mechanical engineering from ChungAng, South Korea.

His research interests include haptic simulation with nonlinear finite element method (FEM) deformation

E-mail: yongwons@buffalo.edu 